\title{
Accumulation of Vitronectin in Atherosclerotic Lesions where Lipids Deposited
}

\section{Ryuichiro Sato, Masahiro Mori, Tsuneo Imanaka, and Tatsuya Takano}

Department of Microbiology and Melecular Pathology, Faculty of Pharmaceutical Sciences, Teikyo University, Sagamiko, Kanagawa, Japan.

A characteristic of atherosclerosis is the accumulation of large amount of lipid, mainly cholesteryl ester, in the arterial wall. These lipids accumulate in the extracellular space or in the cytoplasm of lipid-laden cells which
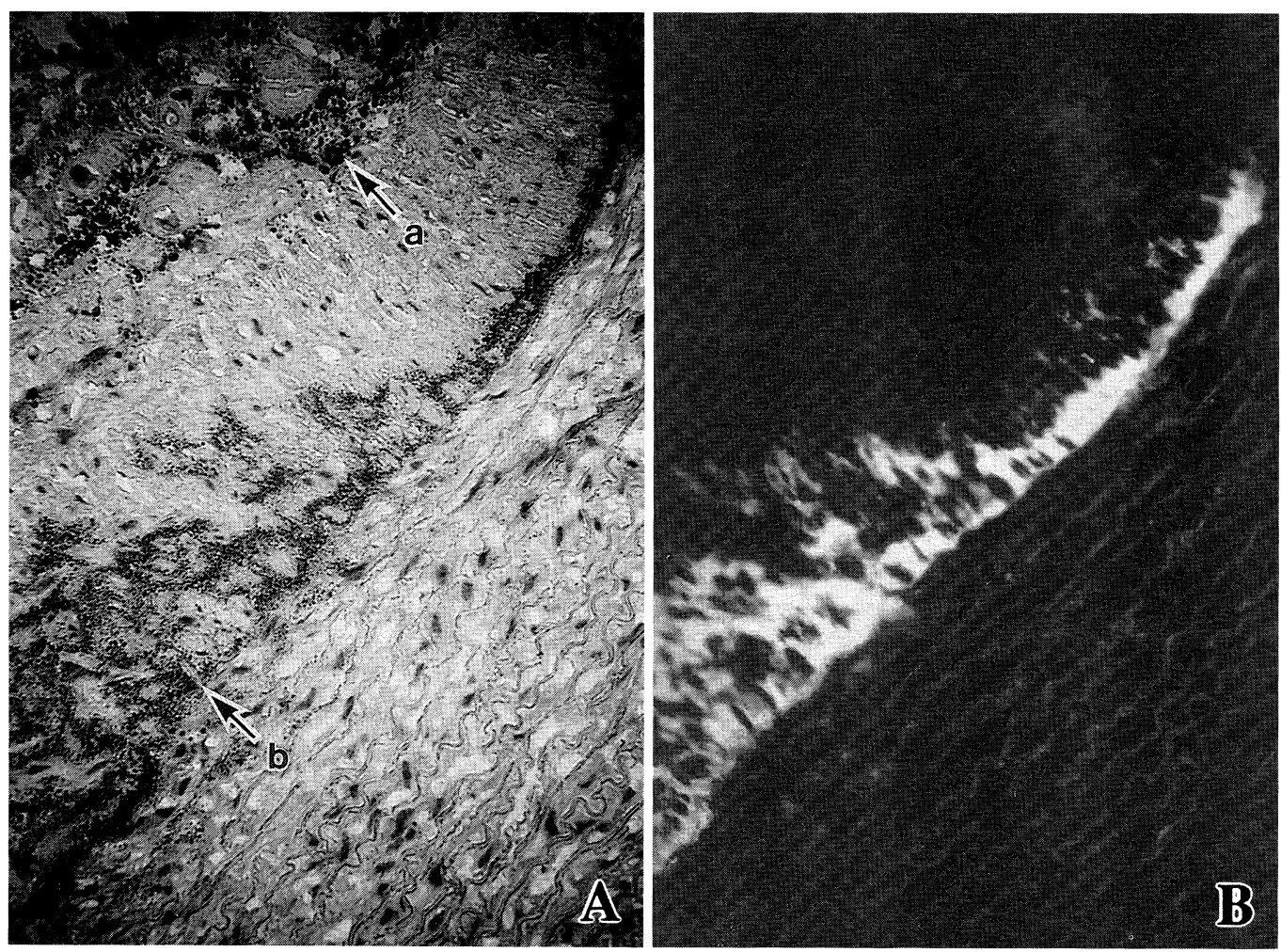

Fig. 1. Correlation of EMR1a/212D antibody staining and lipid deposits. A : Lipid deposits in atherosclerotic lesions (Oil-red $O$ and hematoxylin staining; $\times 200$ ). Two major areas of lipid deposits are observed in atherosclerotic lesions by Oil-red $\mathrm{O}$ staining. Large droplets (arrow a) are observed in the cytoplasm of foam cells and small scattered droplets (arrow b) near the elastic internal lamina are lipid-deposits in the extracellular matrix. B : Immunofluorescence on EMR1a/212D antibody staining is restricted to the extracellular regions with lipid deposits (Indirect immunofluorescent staining of the section adjacent to that for $A$, $\times 200$ ).

This article was presented at the Winter 1991 meeting of the Japan Atherosclerosis Society in Tokyo. 


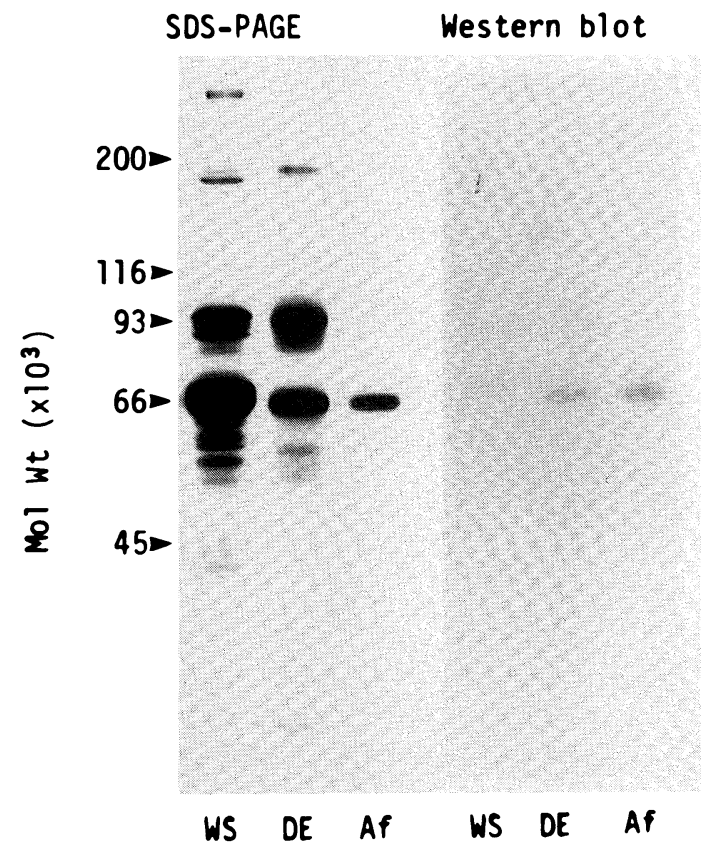

Fig. 2. SDS-PAGE and Western blot analysis of antigenic material at each step of purification. Antigenic material was purified by DEAE Sepharose CL-6B and immunoaffinity chromatography. Antigenic fractions were collected and subjected to electrophoresis in 10\% polyacrylamide gel and stained with silver stain procedure (SDS-PAGE). The nitrocelluose sheet transferred from the gel was developed with the EMR1a/212D monoclonal antibody. Lane WS: WHHL rabbit serum. Lane DE: Peak from DEAESepharose CL-6B. Lane Af: Peak from EMR1a/212Dcoupled Sephoarse 4B. Molecular weight standards were myosin (220kD), $\beta$-galactosidase (116kD). phosphorylase $B$ (93kD), bovine serum albumin $(66 \mathrm{kD})$, and ovalbumin $(45 \mathrm{kD})$.
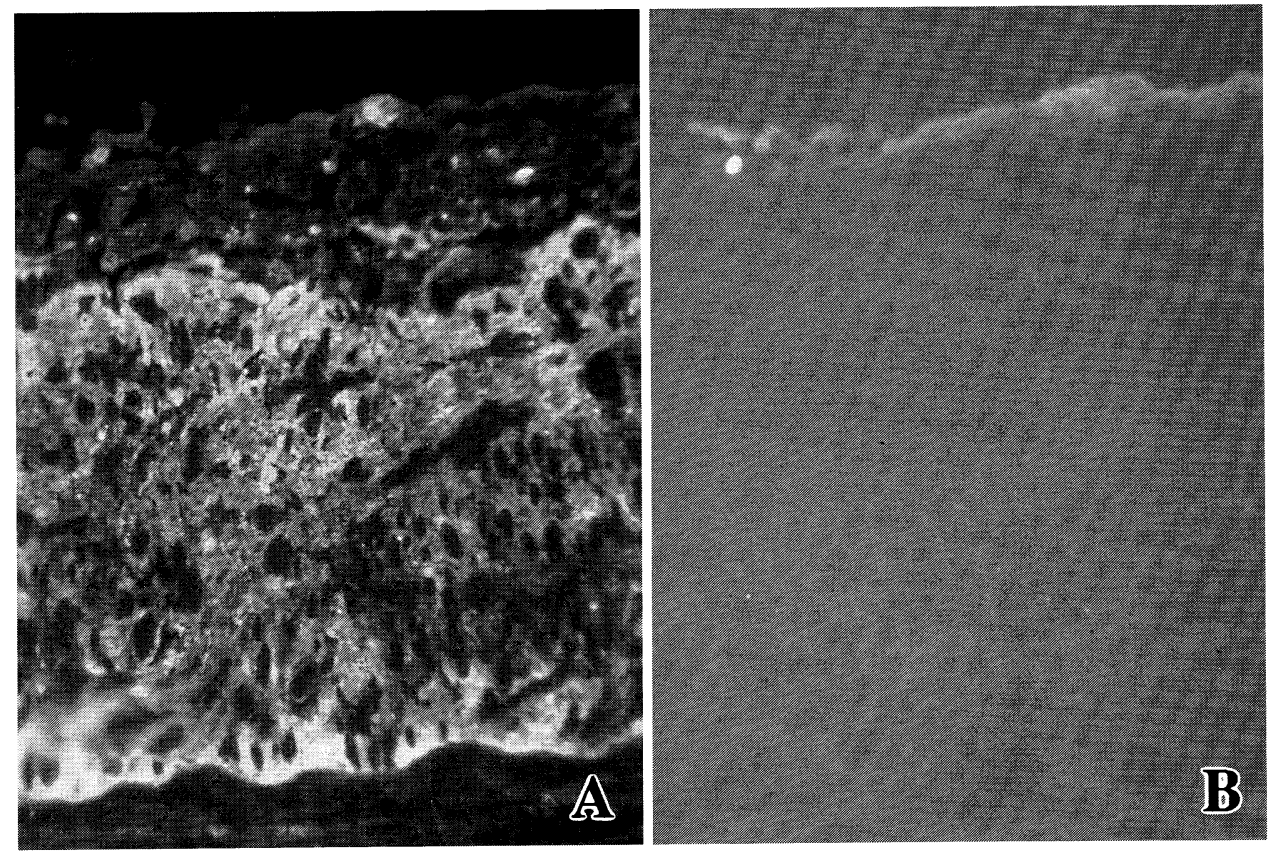

Fig. 3. Effect of neuraminidase on the antigenic activity of tissue section of atherosclerotic aorta. A : Immunofluorescence on EMR1a/212D monoclonal antibody staining is observed in the extracellular matrix of atherosclerotic lesions (Indirect immunofluorescence staining: $\times 200)$. B : After the incubation with neuraminidase $(10 \mathrm{mU} /$ $\mathrm{ml}$ ) at $37^{\circ} \mathrm{C}$ for 24 hours immunofluorescence on the section adjacent to that for $A$ disappeared (Indirect immunofiuorescence staining: $\times 200$ ). 

G Q term

CCCTCAGCTAGAGGCTCTGTCATGGCACCTCTGAGACCCATCTTCACGCTGGCTCTGCTGCTGTGGGTTGTTCTGGCTGACCAAGAGTCC $\begin{array}{llllllllllllllllllllllll}M & A & P & \text { L } & R & P & I & F & T & \text { L } & A & \text { L } & \text { L } & \text { L } & \text { W } & \text { V } & \text { V } & \text { L } & \text { A } & \text { D } & Q & E & S\end{array}$ TGCAAGGACCGCTGCACGGAGGGCTTCAACGCCAACAGGAAGTGTCAGTGTGATGAGCTCTGCTCCTACTACCAGAGCTGCTGCGCTGAC

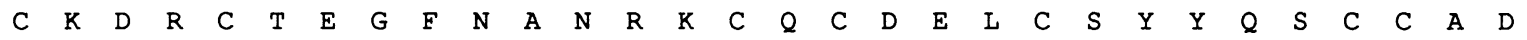
TACGCGGCCGAGTGCAAGCCCCAAGTGACCCGCGGGGACGTGTTCACTATGCCGGAAGATGAATATGGGCCCTATGACTACATCGAGCAG

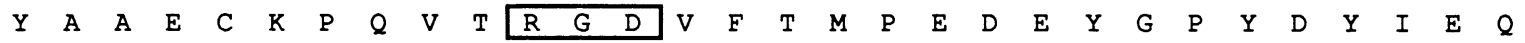
ACCAAAGACAATGCCAGCGTCCATGCACAGCCCGAGAGCCCCACCGTGGGCCAAGAGCCAACCCTGAGCCCTGACCTGCAGACTGAGGGG

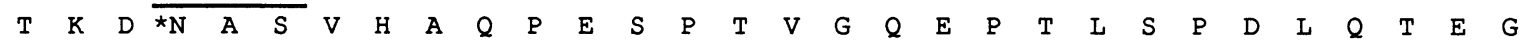
GGCGCTGAGCCTACACACGAGGTCCCCCTGGAGCCTGAGATGGAGACCTTAAGGCCTGAAGGGGAGGACCTGCAGGCCGGGACCACGGAG $\begin{array}{lllllllllllllllllllllllllllllllllllllllll}G & A & E & P & T & H & E & V & P & L & E & P & E & M & E & T & L & R & P & E & G & E & D & L & Q & A & G & T & T & E\end{array}$ CTAGGGACATCGGCGTCCCCAGCAGAGGAGGAGCTGTGCAGTGGGAAGCCTTTTGACGCCTTCACCGACCTCAAGAATGGTTCCCTCTTT

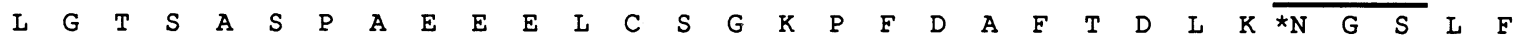
GCTTTCCGAGGACAGTACTGCTACGAGCTAGACGAAACGGCAGTGAGGCCCGGGTACCCCAAGCTCATCCAGGACGTCTGGGGTATTGAG $\begin{array}{lllllllllllllllllllllllllllllllllllll}A & F & R & G & Q & Y & C & Y & E & L & D & E & T & A & V & R & P & G & Y & P & K & L & I & Q & D & V & W & G & I & E\end{array}$ GGCCCCATTGACGCTGCCTTCACCCGCATCAACTGCCAGGGCAAGACCTACCTCTTCAAGGGCAGCCAGTACTGGCGCTTCGAGGACGGT

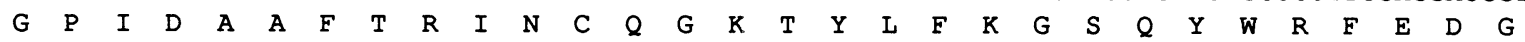
ATCCTGGACCCTGACTACCCTCGCAACATCTCTGAAGGCTTCAGTGGCATCCCAGACAACGTGGATGCGGCCTTTGCCCTCCCTGCTCAC

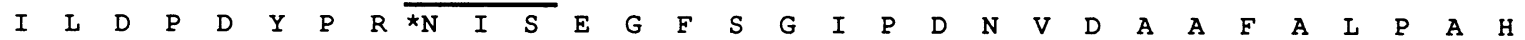
AGCTACAGTGGCCGGGAGCGGGTCTACTTCTTCAAGGGGGATAAGTACTGGGAGTACCAGTTCCAGCAGCAGCCCAGTCAGGAGGAGTGT $\begin{array}{lllllllllllllllllllllllllllllllllll}S & Y & S & G & R & E & R & V & Y & F & F & K & G & D & K & Y & W & E & Y & Q & F & Q & Q & Q & P & S & Q & E & E & C\end{array}$ GAGGGCAGCTCCCTGTCGGCCGTGTTTGAGCACTTCGCCATGCTGCATCGGGACAGCTGGGAGGACATCTTCAAGCTTCTCTTCTGGGGC

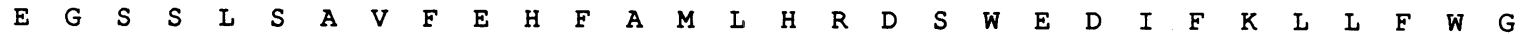
AGACCCTCTGGTGGTGCCAGACAGCCCCAGTTCATCAGCCGGGACTGGCACGGGGTGCCCGGGAAAGTGGATGCAGCCATGGCTGGCCGC $\begin{array}{llllllllllllllllllllllllllllll}R & P & S & G & G & A & R & Q & P & Q & F & I & S & R & D & W & H & G & V & P & G & K & V & D & A & A & M & A & G & R\end{array}$ ATCTACATCTCGGGCTTGACGCCCAGCCCCTCTGCAAAGAAGCAAAAATCTAGGCGTCGCAGCCGCAAACGCTACCGCTCCCGCTATGGC

\begin{tabular}{lllllllllllll|lllllllllllllllllll}
$I$ & $Y$ & $I$ & $S$ & $G$ & $L$ & $T$ & $P$ & $S$ & $P$ & $S$ & $A$ & $K$ & $K$ & $Q$ & $K$ & $S$ & $R$ & $R$ & $R$ & $S$ & $R$ & $K$ & $R$ & $Y$ & $R$ & $S$ & $R$ & $Y$ & $G$ \\
\hline
\end{tabular} CGTGGCCGTAGCCAAAACTCCCGCCGGCTGTCCCGTTCAATCTCACGGTTATGGTTCTCCAGCGAGGAGGTCTCGCTGGGGCCCTACAAC

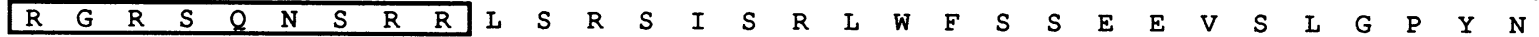
TATGAGGACTACGAGACGAGCTGGCTCAAGCCTGCCACCTCGGAGCCCATCCAGAGCGTCTACTTCTTTTCAGGAGACAAGTACTACCGA $\begin{array}{lllllllllllllllllllllllllllllllllllll}Y & E & D & Y & E & T & S & W & L & K & P & A & T & S & E & P & I & Q & S & V & Y & F & F & S & G & D & K & Y & Y & R\end{array}$ GTCAACCTCCGCACACAGCGAGTGGACACTGTGAACCCCCCCTATCCCCGCTCCATTGCCCAGTACTGGCTCGGCTGCCCAGCCCCTGGC $\begin{array}{lllllllllllllllllllllllllllllllllll}V & N & I & R & T & Q & R & V & D & T & V & N & P & P & Y & P & R & S & I & A & Q & Y & W & L & G & C & P & A & P & G\end{array}$

Fig. 4. Nucleotide sequence and predicted amino acid sequence of GP66 cDNA. The nucleotide sequence was determined by the dideoxynucleotide chain terminal method. Single-letter amino acid symbols are shown under the nucleotide sequence. The cell attachment site (Arg-Gly-Asp) and the glycosaminoglycan binding site are boxed. Possible $\mathrm{N}$-linked glycosylation sites are indicated by an asterisk. The initiation codon (ATG) and polyadenylation signal, AATAAA, at the $3^{\prime}$ end are underlined. derived from either macrophages or modified smooth muscle cells. Possible sources of the lipids deposited in the extracellular space include both the accumulation of plasma lipoproteins and release of lipids from ruptured lipid-laden cells. However, the precise mechanism by which these lipids accumulate in arterial wall during the development of atherosclerosis is poorly understood.

For the first step to understand the mechanism, we have produced monoclonal antibodies to characterize macromolecules enriched in atherosclerotic lesions using a crude homogenate of atherosclerotic aorta from Watanabe-heritable hyperlipidemic (WHHL) rabbits as a complex mixture of immunogens. On selection of specific antibodies by indirect immunohistochemical staining of frozen sections of atherosclerotic aorta, we obtained a monoclonal antibody (EMR1a/212D) which recognized the extracellular matrix where lipids deposited (Fig. 1) (1).

Since antigenic activity was detected in serum of WHHL rabbits, we attempted to purify the antigen from the
Table 1. $\mathrm{NH}_{2}$-terminal sequences of GP66 and human vitronectin.

$$
\begin{aligned}
& \text { G: Asp-Gln-Glu-Ser-?-Lys-Asp-Arg }{ }^{8}- \\
& \text { H: Asp-Gln-Glu-Ser-Cys-Lys-Gly-Arg- } \\
& \text { G: ?-Thr-Glu-Gly-Phe-Asn-Ala-Asn }{ }^{16}- \\
& \text { H: Cys-Thr-Glu-Gly-Phe-Asn-Val-Asp- } \\
& \text { G: Arg-Lys-?-Gln-?-Asp-Glu-Leu }{ }^{24}- \\
& \text { H: Lys-Lys-Cys-Gln-Cys-Asp-Glu-Leu- }
\end{aligned}
$$

G ; GP66 purified by DEAE-Sepharose CL-6B and immunoaffinity chromatography was subjected to SDSPAGE and then electroblotted onto polyvinylidene difluoride membrane was analyzed with the Applied Biosystems model 477A Pulse Liquid Phase Sequencer. Since cysteines in the protein were not modified, they were not recovered in phenylthiohydantoin derivatives.

$\mathrm{H}$; human vitronectin reropted by Suzuki et al. (3) 
serum (2). Using a DEAE-Sepharose CL-6B column, an EMR1a/212D coupled immunoaffinity column, a single band was obtained on SDS-PAGE (Fig. 2). The purified antigenic material was a $66 \mathrm{kD}$ glycoprotein (GP66) with characteristics differ from other types of macromolecules known to be found in atherosclerotic plaques. The neuraminidase-treated (Fig. 3) or trypsin treated GP66 lost its antigenic activity, suggesting that sialic acid is one part of the epitope and that the polypeptide backbone around it also participates in it.

The amino acid composition of GP66 was analyzed in a Hitachi Model 835 amino acid analyzer. GP66 was rich in GIx and Asx and poor in Arg and Lys (2). The amino acid terminal sequence of GP66 was determined after electroblotting onto polyvinylidene difluoride membrane by a Model 477 A Pulse Liquid Phase Sequencer (Applied Biosystems) (Table 1). Twenty amino acid residues in the first 24 amino acid residues of GP66 were identical to those of human vitronectin, if it is assumed that the 5th, 9th, 19th and 21st amino acid residues of GP66 are cysteine. Using synthetic oligonucleotide probes deduced from the partial amino acid sequences, we isolated cDNA clones from a $\lambda$ gt 10 library derived from Japanese white rabbit liver mRNA. The cDNA insert is 1540 base pairs long, with an open reading frame of 1425 nucleotides coding for a polypeptide of 475 amino acids (Fig. 4) (4). The region consisting of the first 19 amino acids, rich in hydrophobic residues, represents the GP66 signal peptide. The $\mathrm{NH}_{2}$-terminal amino acids deduced from nucleotide sequencing were identical to those determined by protein sequencing. The mature GP66 contained the somatomedin B sequence (residues 1-44), a cell attachment site (residues 45-47), three potential $\mathrm{N}$-linked glycosylation sites and the expected glyco- saminoglycan binding sites (residues 347-373) (Fig. 4). There is a $76 \%$ amino acid sequence homology with human vitronectin.

To determine the number of $\mathrm{N}$-linked oligosaccharide chains in GP66, the glycoprotein was partially digested by $\mathrm{N}$-glycanase by incubating for various time periods. In addition to intact GP66, three bands appeared in succession on SDS-PAGE during the $24 \mathrm{~h}$ incubation period. This result is in agreement with the finding from the CDNA nucleotide sequence that GP66 has three potential Nlinked glycosylation sites.

To compare GP66 with rabbit vitronectin, we purified rabbit vitronectin by heparin-binding column chromatography. Rabbit vitronectin and GP66 migrated identically on SDS-PAGE and were also recognized by EMR1a/212D (Fig. 5). We have found that GP66, as well as commercial human vitronectin, promoted Madin-Darby canine kidney cell spreading and also had a heparin-binding activity. These results strongly suggest that GP66 is rabbit vitronectin.

The size of GP66 mRNA and the expression of GP66 gene in liver of normal and WHHL rabbits were examined by Northern and dot-blot hybridizations. The results shown in Fig. 6 indicate one-transcript in RNA from normal and $\mathrm{WHHL}$ rabbit liver and the level of GP66 mRNA in WHHL rabbit was very similar to that in normal rabbit. To elucidate the relationship between the amount of GP66 in plasma and atherosclerotic aorta, dot-immunobinding assay was conducted. There was no difference in the concentration of GP66 in normal and WHHL plasma. However, in contrast, the amount of GP66 in the arterial wall of WHHL rabbit was 9-fold higher than that of normal rabbit (Table 2). The increase in GP66 content was comparable to the augmentation in total cholesterol con-

\section{SDS-PAGE Western blot}

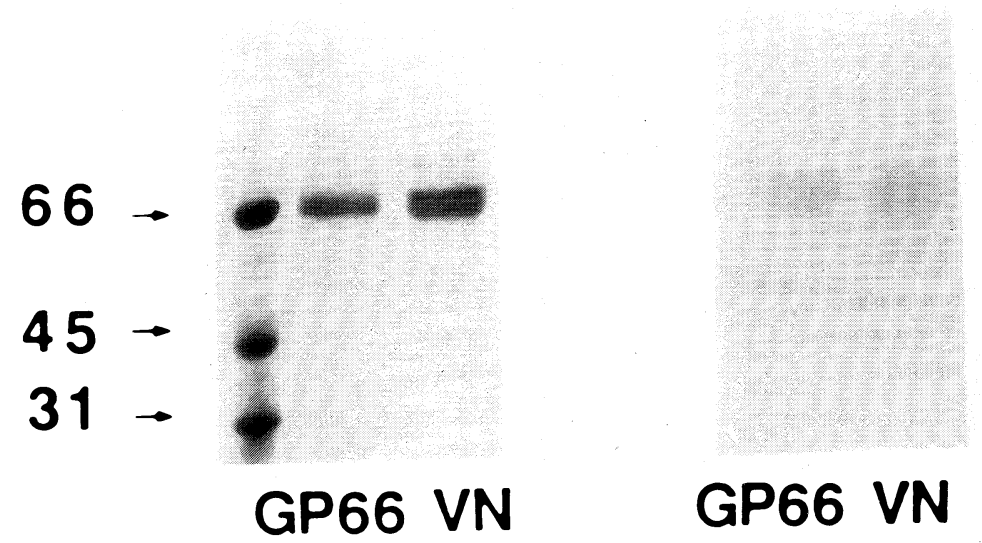

Fig. 5. Demonstration of immunological identity of GP66 and rabbit vitronectin. GP66 (lanes 1 and 3 ) and rabbit vitronectin (lanes 2 and 4) purified by the method of Yatohgo et al. [5] were analyzed on $10 \%$ SDS-PAGE (A) and immunoblotted with EMR1a/212D (B). The gel was stained by the silver stain procedure $(A)$. Molecular mass $(\mathrm{KD})$ of protein standards are indicated at the left of the gel. 
A

$285-$

185

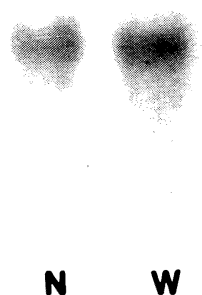

Fig. 6. RNA gel-blot $(A)$ and dot-blot (B) hybridization analysis of GP66 mRNA expression in normal $(\mathrm{N})$ and WHHL (W) rabbit liver. GP66 CDNA probe was hybridized with total RNA isolated from liver. A : $10 \mu \mathrm{g}$ of RNA were electrophoresed on $0.8 \%$ agarose/formaldehyde gels and transferred onto nyloy filters. The size markers are the $28 \mathrm{~S}$ and $18 \mathrm{~S}$ ribosomal RNAs. B : 5 , 2.5 , and $1.25 \mu \mathrm{g}$ of RNA were immobilized on nylon filters.

tent in arterial wall and plasma.

These results suggest that the accumulation of GP66 in atherosclerotic lesions is not the results of an increase in plasma GP66. There are a number of possible explanations. First, GP66 is introduced into the arterial wall as a result of arterial injury, becoming bound to glycosaminoglycan, collagen or its receptor on the cell surface. An alternative explanation could be that the cells of the vessel wall secrete GP66 into the extracellular space in response to lipid deposition and/or stimulated platelets which reaches high local concentration in atherosclerotic lesions release GP66.

Vitronection is a multifunctional protein promoting attachment and spreading of a variety of cells and interacting with the C5b-7 complement complex. The thrombin anti-thrombin III complex and the plasminogen activator inhibitor-I (6). Some of these functions may be involved in the development of atheroscleotic lesions.

\section{References}

(1) Kimura J, Nakagami K, Amanuma K, Ohkuma S, Yoshida
Table 2. Comparison of the contents of GP66 and total cholesterol in plasma and thoracic arterial wall of normal and $\mathrm{WHHL}$ rabbits.

Four-cm segments of the descending thoracic aorta were removed from the aortic arch and homogenized. Plasma and the supernatant of the aorta homogenate $(12,000 \mathrm{rpm} \times 5 \mathrm{~min})$ were dotted onto nitrocellulose filters. The amount of GP66 was determined using EMR1a/212D and ${ }^{125} \mathrm{I}$-labeled goat anti-mouse IgG. Total cholesterol in plasma and the supernatant of the homogenate was determined by enzymatically. Protein in the supernatant was determined using BCA protein assay reagent. The statistical significance of differences between observed values for normal and WHHL rabbits was tested by Student's $t$ test.

\begin{tabular}{lcc}
\hline & Plasma & Aorta \\
\cline { 2 - 3 } & \multicolumn{2}{c}{$\mathrm{GP66}$} \\
\hline Normal rabbit & $\mu \mathrm{g} / \mathrm{ml}$ & $\mathrm{ng} / \mathrm{mg}$ tissue protein \\
WHHL rabbit & $253 \pm 14^{\mathrm{a}}$ & $4.1 \pm 0.9^{\mathrm{b}}$ \\
\hline & \multicolumn{2}{c}{ Total cholesterol } \\
\hline & $\mathrm{mg} / \mathrm{dl}$ & $\mu \mathrm{g} / \mathrm{mg}$ tissue protein \\
Normal rabbit & $44 \pm 8^{\mathrm{a}}$ & $18 \pm 4^{\mathrm{b}}$ \\
WHHL rabbit & $923 \pm 63^{\mathrm{a}, \mathrm{c}}$ & $259 \pm 13^{\mathrm{b}, \mathrm{c}}$
\end{tabular}

a Values refer to mean + S.E. of five normal rabbits or fou WHHL rabbits.

b Values refer to mean \pm S.E. of three rabbits.

c Represents $\mathrm{P}<0.01$

$\mathrm{Y}$, and Takano $\mathrm{T}$ : Monoclonal antibodies recognizing lipid-laden cells and extracellular regions with lipiddeposits in atherosclerotic aorta. Virchows Arch A, 410 : 159-164, 1986

(2) Nakagami K, Shimazaki O, Sato R, Komine Y, Ohkuma S, and Takano T: Monoclonal antibody EMR1a/212D recognizing site of deposition of extracellular lipid in atherosclerosis. Am J Pathol, 135: 93-100, 1989

(3) Suzuki S, Oldberg A, Hayman EG, Pierschbacher MD, and Ruoslahti $\mathrm{E}$ : Complete amino acid sequence of human vitronectin deduced from cDNA. Similarity of cell attachment sites in vitronection and fibronectin. EMBO J, 4 : 2519-2524, 1985

(4) Sato R, Komine Y, Imanaka T, and Takano T: Monoclonal antibody EMR1a/212D recognizing site of deposition of extracellular lipid in atherosclerosis. J Biol Chem, 265: 21232-21236, 1990

(5) Yatohgo $T$, Izumi $M$, Kashiwagi $H$, and Hayashi $M$ : Novel purification of vitronectin from human plasma by heparin affinity chromatography. Cell Struct Funct, 13: 281292, 1988

(6) Preissner KT: Structure and biological role of vitronectin. Ann Rev Cell Biol, $7: 275-310,1991$ 